\title{
Spontaneous Rupture of a Giant Hepatic Hemangioma-Report of a Case
}

\author{
Shahana Gupta • Vikash Agarwal • \\ Anadi Nath Acharya
}

Received: 27 June 2009 / Accepted: 28 August 2009 / Published online: 21 May 2011

(C) Association of Surgeons of India 2011

\begin{abstract}
Hemangioma is the commonest benign hepatic neoplasm. Most cases are asymptomatic. Spontaneous rupture is rare (1-4\%). Only 34 cases have been reported in adults. None had history of trauma. We report a case, the first from India of spontaneous rupture of a giant hepatic hemangioma, in a 25 year old male presenting with acute abdomen. He underwent right hepatectomy. Histopathology suggested cavernous hemangioma.
\end{abstract}

Keywords Spontaneous rupture $\cdot$ Hepatic hemangioma

\section{Introduction}

Hemangioma is the commonest liver neoplasm in adults as well as in children (prevalence $1-20 \%$ in general population) $[1,2]$.Complications are observed mostly in large hemangioma and include alteration in coagulation (Kasabach-Merrit syndrome) [3] and internal architecture (eg. inflammation), infection in the form of abscess [4], cardiac failure (secondary to arteriovenous shunt of large lesions have been reported in children) [2], compression of adjacent structures and spontaneous rupture.

\footnotetext{
S. Gupta $(\bowtie) \cdot$ V. Agarwal $\cdot$ A. N. Acharya

Department of Surgery,

Institute of Postgraduate Medical Education \& Research,

A.J.C. Bose Road,

Kolkata, India

e-mail: shahanagupta@yahoo.co.in
}

PubMed Medline search identifies 34 cases of spontaneous rupture of hepatic hemangioma in adults ( $>14$ year age) without a history of trauma [5-7].

We report a case, the first in India, of spontaneous rupture of a giant hepatic hemangioma.

\section{Case Report}

A 25 year old male presented to the ER with sudden onset severe diffuse abdominal pain for 5 days without vomiting, absolute constipation or history of recent trauma. He complained of recurrent attacks of vague upper abdominal pain, relieved with analgesics.

On examination, his vitals were stable, abdomen showed signs of peritonitis with mild abdominal distension. Ultrasonography (USG) of abdomen showed presence of hemoperitoneum and an irregular heterogeneous space occupying lesion in the right lobe of liver. Quadrant puncture showed frank blood. Hemoglobin was 8 gram\%.

On laparotomy through midline incision, a hemangiomatous mass(size: approximately $9 \times 12 \mathrm{~cm}$ ) was found in right lobe of liver with significant hemoperitoneum. No obvious breach in capsule was visible, though there was oozing from a few points (Fig. 1).

Oozing was stopped by perihepatic packing(with large abdominal packs for 15-20 min) and application of local hemostatic agents (oxidized regenerated cellulose and gelfoam). A subhepatic drain was placed. Postoperative period was uneventful. At discharge, his Liver Function 


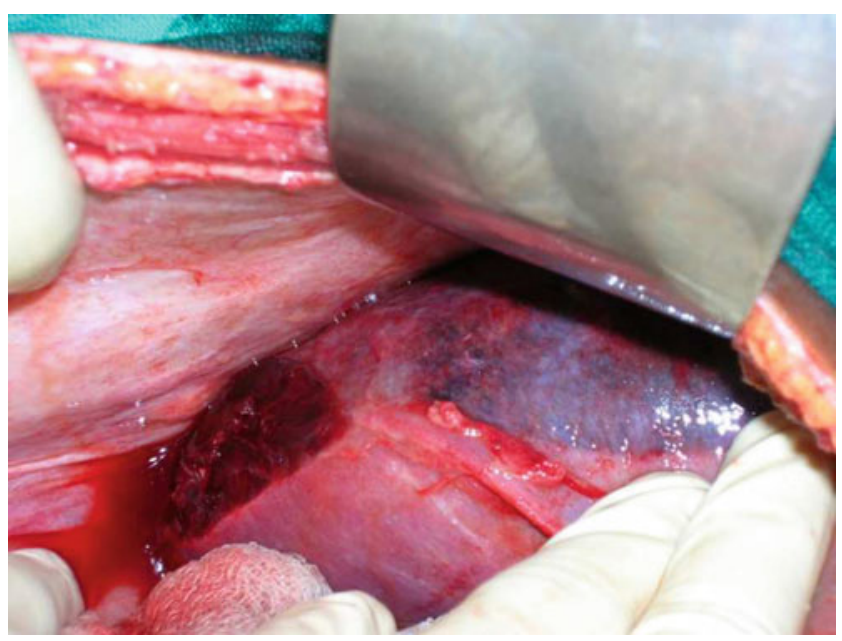

Fig. 1 Hemangiomatous mass in right lobe of liver

Test (LFT), Prothrombin Time (P-Time) and platelet counts were normal.

At follow-up, contrast-enhanced CT (CECT) scan of abdomen revealed a thick-walled mixed-density lesion in segments V, VII and VIII of liver (size $9 \times 12.84 \times 9.27 \mathrm{~cm} 3$ ), non-enhancing on contrast. A mixed density subcapsular collection was detected with a capsular breach in posterior wall of the lesion (Fig. 2).

The patient underwent right hepatectomy after 2 months through a J-shaped incision(vertical limb extending from xiphoid to a point near midpoint between xiphoid and umbilicus; transverse limb extending to mid-axillary line

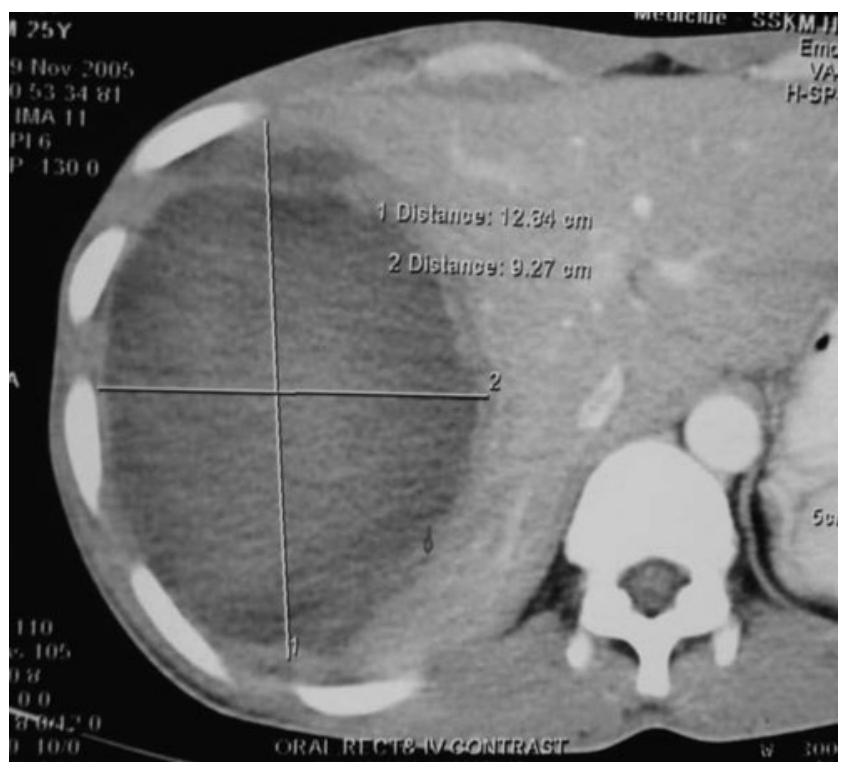

Fig. 2 CECT abdomen showing the hemangioma and breach in capsule posteriorly parallel to right costal arch). Pringle's manouver was utilized for vascular control.

Postoperative period was uneventful. Histopathological examination of specimen suggested cavernous hemangioma.

During follow-up at 2 years, the patient is doing fine with a normal LFT and USG abdomen shows evidence of hepatic regeneration.

\section{Discussion}

Hemangioma is the commonest benign neoplasm of the liver. Hemangioma $>4 \mathrm{~cm}$ in diameter is referred to as a GIANT hemangioma. Its pathogenesis is not well understood. Oestrogen may have a role $\left[1^{\mathrm{a}}, 8\right]$.

Most hemangioma are asymptomatic, detected incidentally on imaging of abdomen for unrelated reasons [8]. Pain related to an uncomplicated hemangioma is usually due to associated disorders e.g., peptic ulcer. A large hemangioma may present as an abdominal mass or pain. Most hemangioma remain stable in size $\left[1^{\mathrm{a}}\right]$ or show minimal increase over time [9]. Laboratory investigations including LFT are normal. Of the complications, spontaneous rupture, a rare event $(1-4 \%)$ is associated with high mortality rate (60-75\%).

Most hemangioma are diagnosed accurately on imaging (CECT, MRI, USG). Strict diagnostic criteria on CT Scan are described $\left[1^{\mathrm{b}}\right]$ but variations exist. Large hemangiomas appear heterogeneous with marked central areas corresponding to thrombosis, extensive hyalinisation and fibrosis. Very slow filling hemangiomas appear as hypoattenuating lesions on multiphasic examination and may not have classic globular enhancement,as is observed in this case. Hemangioma developing in abnormal liver are difficult to diagnose. MRI is helpful in such cases.

Hemangiomas are no longer considered a contraindication to needle biopsy. No serious complications were observed in two large series $\left[1^{c}\right]$. Percutaneous biopsy should be restricted to atypical cases only. For uncertain diagnosis, laparoscopy with contact ultrasound is effective $\left[1^{\mathrm{c}}\right]$.

Whatever be the size, there is no treatment for asymptomatic hemangioma. The patient must be assured about rare occurrence of growth and minimal risk of complications $\left[1^{\mathrm{c}}\right]$. Indications for surgery are severe symptoms, complications (e.g., intractable cardiac failure and/or refractory consumptive coagulopathy, rupture) and inability to exclude malignancy $[2,10]$. Surgical resection is the definitive treatment $\left[1^{\mathrm{d}}, 11\right]$. Choice of excision (Enucleation, hepatic resection, laparoscopic techniques) depends on the size and anatomic location of the lesion. Enucleation is associated with fewer complications and allows preservation of maximal amounts of liver tissue. Use 
of Cavitron Ultrasonic Surgical Aspirator (CUSA) facilitates dissection. Preoperative transcatheter arterial embolisation (TAE) reduces hemorrhage making resection safer. Radiation therapy and hepatic artery ligation are less effective options. Liver transplantation is an option for technically unresectable complicated giant hemangioma $\left[1^{\mathrm{d}}\right]$.

The most recent of the 34 published reports of spontaneous rupture appeared in 2008 [7]. In a review of 27 cases [5] which includes 17 females and 9 males, (age 15-73 years, average 48.2), lesion was found in right lobe in 14 , in left in 10 , in both lobes in 1 . Tumour size range from $3 \mathrm{~cm}$ to the whole of right hepatic lobe; 16 of 19 tumours of known size were giant hemangiomas (mean diameter $14.8 \mathrm{~cm}$, range $6-25 \mathrm{~cm}$ ). Of 27 patients, 4 died of hemorrhagic shock without surgery. Twenty-two underwent surgery of whom 13 were resected, 5 sutured and 4 underwent tamponade. Of the 13 patients who underwent a resection, 3 died. Four patients underwent TAE prior to an elective hepatic resection without any operative mortality. 2 of 5 sutured patients and 3 of 4 patients who underwent tamponade died. 8 of 22 patients treated surgically died. In a report [6] published after this review appeared, the patient was treated successfully with emergency hepatic bisegmentectomy.

A successful conservative management of spontaneous rupture of hemangioma with hepatic arterial embolisation alone, has been reported [7]. The authors opine that this is a safe treatment in patients with hemodynamic stability or with initial hemodynamic instability responding to fluid resuscitation. In cases of recurrent hemorrhage,delayed surgery is suggested.

\section{References}

1. Blumgart LH (2007) Surgery of the Liver, Biliary tract and Pancreas, vol. 2, Chap 70; 4th ed,; page $1131^{\mathrm{a}} ; 1132-33^{\mathrm{b}} ; 1134^{\mathrm{c}}$; $1135^{\mathrm{d}}$.

2. Costa CM, Rodrigues KE, Campos HG, Gibelli N, Ayoub A, Kayabashi M, Sredni ST, Camargo BD (2000) Hepatic Hemangioma. J Pediatr(Rio J) 76(1):87-90

3. Valdes MM, Ortiz Sanchez ML, Rodrigo Aqudo JL, Miras Lopez M, Pons Minano JA, Carballo Alvarez F (2008) Giant hepatic hemangioma associated with Kasabach Merrit syndrome. Rev Esp Enferm Dig 100(8):511-513

4. Pinkernelle J, Neumann U, Hanninen EL (2007) An unusual cause of epigastric pain: infected giant liver hemangioma. Acta Radiol 48(2):142-144

5. Corigliano N, Paolo M, Pietro MA, Balducci G, Caterino S, Ramacciato G, Ziparo V (2003) Hemoperitoneum from a spontaneous rupture of a Giant Hemangioma of the Liver: Report of a case. Surg Today 33:459-463, and Reference 12 cited therein

6. Griffa B, Basilico V, Bellotti R, Griffa A, Senatore S, Capriata G (2005) Spontaneous rupture of a giant subcapsular hemangioma of the liver with hemoperitoneum and hemorrhagic shock: a case report. Chir Ital 57(3):389-392

7. Vokaer B, Kothonidis K, Delatte P, De Cooman S, Pector JC, Liberale G (2008) Should ruptured liver hemangioma be treated by surgery or by conservative means? A case report. Acta Chir Belg 108(6):761-764

8. Trotter JF, Everson GT (2001) Benign focal lesions of the liver. Clin Liver Dis 5:17-42

9. Nghiem HV, Bogost GA, Ryan JA, Lund P, Freeny PC, Rice KM (1997) Cavernous hemangiomas of the liver-enlargement over time. AJR Am J Roentgenol 169:137-141

10. Yoon SS, Charny CK, Fong Y, Jarnagin WR, Schwartz LH, Blumgart LH, DeMatteo RP (2003) Diagnosis, management and outcomes of 115 patients with hepatic hemangioma. J Am Coll Surg 197:392-402

11. Demircan O, Demiryurek H, Yagmur O (2005) Surgical approach to symptomatic giant cavernous hemangioma of the liver. Hepatogastroenterology 52(61):183-186 